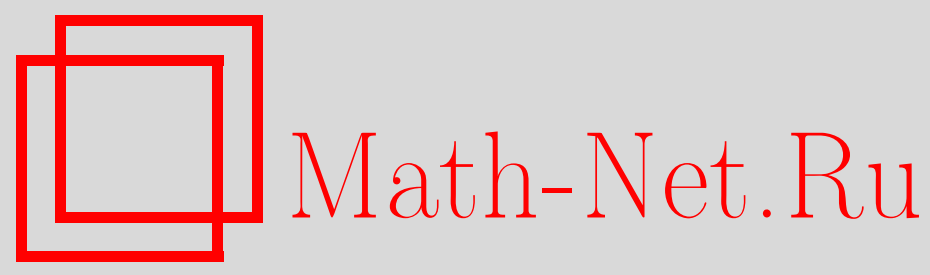

S. V. Agievich, Режим счетчика с шифрованием синхропосылок и его расширение до аутентифицированного шифрования, Mатем. вопр. криптогр., 2020, том 11, выпуск 2, 724

DOI: https://doi.org/10.4213/mvk318

Использование Общероссийского математического портала Math-Net.Ru подразумевает, что вы прочитали и согласны с пользовательским соглашением http://www.mathnet.ru/rus/agreement

Параметры загрузки:

IP: 18.207 .199 .55

26 апреля 2023 г., 03:41:57

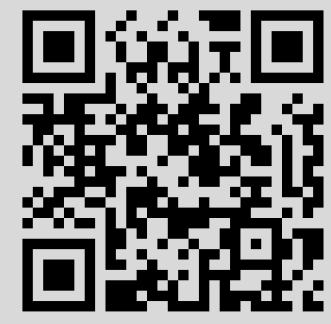




\title{
The Counter mode with encrypted nonces and its extension to authenticated encryption
}

\author{
S. V. Agievich \\ Research Institute for Applied Problems of Mathematics and Informatics, \\ Belarusian State University, Minsk, Belarus \\ Получено 05.XI.2019
}

Abstract. In the modified CTR (Counter) mode known as CTR2, nonces are encrypted before constructing sequences of counters from them. This way we have only probabilistic guarantees for non-overlapping of the sequences. We show that these guarantees, and therefore the security guarantees of CTR2, are strong enough in two standard scenarios: random nonces and non-repeating nonces. We also show how to extend CTR2 to an authenticated encryption mode which we call CHE (Counter-HashEncrypt). To extend, we use one invocation of polynomial hashing and one additional block encryption.

Keywords: CTR mode, authenticated encryption, block cipher, polynomial hashing, gamma overlapping

\section{Режим счетчика с шифрованием синхропосылок и его расширение до аутентифицированного шифрования \\ С. В. Агиевич \\ НИИ прикладных проблем математики и информатики, Белорусский государственный университет, Минск, Республика Беларусъ}

Аннотация. $\mathrm{B}$ модифицированном режиме CTR (счетчика), известном как CTR2, синхропосылки зашифровываются перед тем, как по ним строятся последовательности счетчиков. Гарантии неперекрытия последовательностей становятся вероятностными. Показано, что эти гарантии, а следовательно, и гарантии стойкости CTR2, достаточно высоки в двух стандартных сценариях: случайные синхропосылки и неповторяющиеся синхропосылки. Предложен способ расширения CTR2 до режима аутентифицированного шифрования Counter-Hash-Encrypt (CHE). В CHE дополнительно к CTR2 требуются одно полиномиальное хэширование и одно зашифрование блока.

Ключевые слова: режим CTR, аутентифицированное шифрование, блочный шифр, полиномиальное хэширование, перекрытие гамм 


\section{Preliminaries}

Let $E$ be a block cipher with block size $n$ and key space $\mathcal{K}$. It is a multiset consisting of permutations $E_{K} \in \operatorname{Perm}(n)$ which are indexed by secret keys $K \in \mathcal{K}$. Here $\operatorname{Perm}(n)$ is the set of all permutations over $\{0,1\}^{n}$. Elements of $\{0,1\}^{n}$ are called blocks. Let $N=2^{n}$ denote their number.

We also denote by $\{0,1\}^{*}$ the set of all binary words of finite length. For a word $u \in\{0,1\}^{*}$, let $|u|$ denote its length. If $u, v$ are words of the same length, then $u \oplus v$ is their bitwise sum modulo 2 (XOR). For a permutation $\pi \in \operatorname{Perm}(n)$, let $\pi^{i}$ be its $i$ th compositional power $\left(\pi^{0}\right.$ is the identity permutation). Denote by $m^{[i]}$ the $i$ th factorial power of a positive integer $m: m^{[i]}=m(m-1) \ldots(m-i+1)$.

To extend the action of $E$ from $\{0,1\}^{n}$ to $\{0,1\}^{*}$, encryption modes are used. One of the most popular is CTR. In this mode, a unique nonce $S \in\{0,1\}^{n}$ is repeatedly transformed by a public permutation next. The resulting sequence

$$
C_{1}=S, C_{2}=\operatorname{next}\left(C_{1}\right), C_{3}=\operatorname{next}\left(C_{2}\right), \ldots
$$

is encrypted using $E_{K} \in E$ to get the blocks

$$
\Gamma_{1}=E_{K}\left(C_{1}\right), \Gamma_{2}=E_{K}\left(C_{2}\right), \ldots
$$

To encrypt a plaintext $X \in\{0,1\}^{*}$, the first $\lceil|X| / n\rceil$ blocks $\Gamma_{1}, \Gamma_{2}, \ldots$ are used. They are concatenated and then truncated to $|X|$ bits. The resulting word $\Gamma \in\{0,1\}^{|X|}$ is XORed with $X$ to produce the ciphertext

$$
Y=X \oplus \Gamma \text {. }
$$

In the Soviet standard GOST 28147 [7], the word $\Gamma$ is called a gamma. That is why the notations. The blocks $C_{1}, C_{2}, \ldots$ are usually called counters. That is why CTR (CounTeR).

Suppose that in two encryption sessions gammas $\Gamma$ and $\Gamma^{\prime}$ overlap. Then an adversary who has intercepted a plaintext-ciphertext pair $(X, Y)$ in one session can restore $\Gamma=X \oplus Y$ and then partially reconstruct $X^{\prime}$ from $Y^{\prime}=$ $X^{\prime} \oplus \Gamma^{\prime}$ in the parallel session. Thereby, a gamma overlapping is considered as a defect of the CTR encryption.

To avoid overlapping, a permutation next is chosen to have long disjoint cycles in its cycle decomposition. The nonces $S$ of different sessions are picked from different cycles or a new nonce continues the cycle (actually, the sequence of counters) from the previous session. This approach, 
implemented in the standards $[6,8,9]$, ensures that all counters in all sessions are unique. In other words, there are no collisions between counters and gamma overlapping is certainly impossible.

Unfortunately, such strict guarantees of no collisions/non-overlapping force the nonce management to be rather complicated. One has to use a safe monotonous timer to generate nonces or a rewritable memory to store them between sessions. Both options may be difficult to implement in certain cryptographic devices. The third option, random generation of nonces, does not match the approach, at least it is not allowed in the mentioned standards.

Another approach, probabilistic guarantees of gamma non-overlapping, was proposed in GOST 28147 and repeated in [15], where a nonce $S$ is first encrypted and then transformed by next:

$$
C_{1}=\operatorname{next}\left(E_{K}(S)\right),
$$

not $C_{1}=S$. (To be completely accurate, GOST's next is not a permutation: it acts bijectively on only a $2^{n / 2}\left(2^{n / 2}-1\right)$-element subset of $\{0,1\}^{n}, n=64$.) The similar scheme

$$
C_{1}=E_{K}(S)
$$

was considered later by P. Rogaway in [14], where the corresponding encryption mode is called CTR2. We extend this name to the GOST case. It is natural because the main point there is nonce encryption, the optional invocation of next is not essential.

Nonce encryption has obvious drawbacks. First, it slightly decreases the overall effectiveness of the mode. Second, it throws $C_{1}$ at an unpredictable point of next's cycle that may cause a collision with other counters.

On the other hand, the probability of collisions is controllably small under reasonable restrictions on the amount of data processed with a single key. We confirm this fact in Section 2 in terms of a game called "Battleship on a circle".

A control over collisions allows us to prove the security of CTR2 in the CPA (Chosen Plaintext Attack) settings. This is done in Section 3. In a nutshell, we embed well-known or easily derived combinatorial estimates within the context of Provable Security. Surprisingly, despite the long history of CTR2, it seems that we provide the first explicit proof of its security. We examine two techniques for nonce generation: random nonces and nonrepeating nonces. Note that we do not require that the nonce management deterministically ensures uniqueness of all counters in all sessions and thus allow it to be more flexible. 
An additional argument in favor of nonce encryption is that it provides an easy extension of the conventional CTR encryption to authenticated encryption (AE). In Section 4, we show how to construct such an extension using polynomial hashing and one additional invocation of $E_{K}$. We call the resulting scheme CHE, meaning the Counter-Hash-Encrypt cascade. It is actually one of the two AE schemes briefly described in [1]. There the security of only authentication, not encryption, is considered. In this paper, we fill the gap. We also provide a detailed description of $\mathrm{CHE}$.

Usually, in AE schemes based on polynomial hashing (perhaps the most well-known of them is GCM [10]), a data-driven polynomial is evaluated at a secret point which depends only on $K$. In some cases (including GCM), this point may be recovered with the subsequent compromise of all encryption sessions as soon as a nonce $S$ is used twice. A distinctive feature of CHE is that the secret point depends on $S$. Due to this fact, a repetition of nonces in multiple encryption sessions compromises only these sessions without affecting other ones. Thus, CHE provides reasonable security guarantees against nonce-misusing. To the best of our knowledge, stronger guarantees, the so-called full nonce-misuse resistance where only completely identical sessions compromise each other, are only achieved through two passes over data what is difficult to maintain in many scenarios.

Further we assume that next is a full cycle or almost full cycle permutation. In other words, if $M$ is the maximum cycle length of next, then $M \approx N$. Usually, $M=N$ which is achieved by interpreting blocks of $\{0,1\}^{n}$ as integers modulo $N$ and incrementing these integers in next. Another option for next is to interpret $\{0,1\}^{n}$ as the binary field $\mathbb{F}$ of $N$ elements. Let $a$ be a primitive element of $\mathbb{F}$ and $b$ be an arbitrary element. Then the permutation

$$
\text { next: } \lambda \mapsto a \lambda+b
$$

decomposes into a cycle of length $M=N-1$ and a loop at $b /(1-a)$. We use this next in Section 4.

Finally, it should be mentioned that encrypting a nonce $S$ we make the counters $C_{1}, C_{2}, \ldots$ secret. An adversary is not able to reconstruct any input-output pair of $E_{K}$ even after intercepting all the session data $(S, X, Y)$. Blocking direct access to $E_{K}$ complicates attacks to recover $K$, especially statistical and algebraic attacks which usually strongly depend on the complexity of the simplest accessible cryptographic component. 


\section{Battleship on a circle}

"Battleship on a circle" is played by Navy and an adversary. A game field is a circle on which $M$ points numbered from 0 to $M-1$ are placed. Navy deploys ships on the circle concealing their locations. A ship of displacement $r_{i}$ (a positive integer) occupies $r_{i}$ consecutive points. In total, $q$ ships of overall displacement $r(q \leqslant r \leqslant M)$ are deployed. The adversary makes $q$ shots on the ships.

Detailed rules of the game (see Fig. 1 for example):

1) The adversary splits $r$ into a sum $r_{1}+r_{2}+\ldots+r_{q}$ of positive integers and reports $r_{1}, r_{2}, \ldots, r_{q}$ to Navy.

2) Navy deploys ships at random points on the circle. The bow of the $i$ th ship is placed at point $C_{i, 1}$ and the whole ship occupies the segment $C_{i, 1}, C_{i, 1}+1, \ldots, C_{i, 1}+r_{i}-1$ (additive operations are modulo $M$ ). Collisions of the ships, that is, intersections of their segments, may occur. In the case of a collision, Navy loses and capitulates. Let the event $\mathcal{D}_{1}$ mean no collisions.

3) If Navy has not capitulated, then the adversary makes $q$ shots at different points $S_{1}, \ldots, S_{q}$ on the circle. If at least one shot hits a ship, then the adversary wins. If all the shots miss, which is captured by the event $\mathcal{D}_{2}$, then Navy wins.

Further we consider two variants of the game: $G_{1}$ and $G_{2}$.

In $G_{1}$ the ship bows $C_{i, 1}$ are chosen uniformly at random independently of each other. The shot points $S_{i}$ are also chosen uniformly at random with the only restriction that they must be different. In other words, $\left(S_{1}, \ldots, S_{q}\right)$ is a random $q$-permutation of $M$ numbers. There are $M^{[q]}$ ways to choose it.

In $G_{2}$ the bows also form a random $q$-permutation. Shot points are arbitrary distinct.

Let us immediately explain that the games $G_{1}$ and $G_{2}$ simulate attacks on CTR2 with random and non-repeating nonces respectively. Ships correspond to sequences of counters. The lengths of the sequences may be chosen by an adversary who needs only to keep the total length, that is, the total amount of plaintext-ciphertext data. A collision of ships trivially means a gamma overlapping. Shots are more subtle. A hit means that a nonce coincides with one of the internal counters. We will explain further details in the next section.

We are interested in the probability that Navy wins: $\mathbf{P}\left\{\mathcal{D}_{1} \mathcal{D}_{2}\right\}=$ $\mathbf{P}\left\{\mathcal{D}_{2} \mid \mathcal{D}_{1}\right\} \mathbf{P}\left\{\mathcal{D}_{1}\right\}$ 


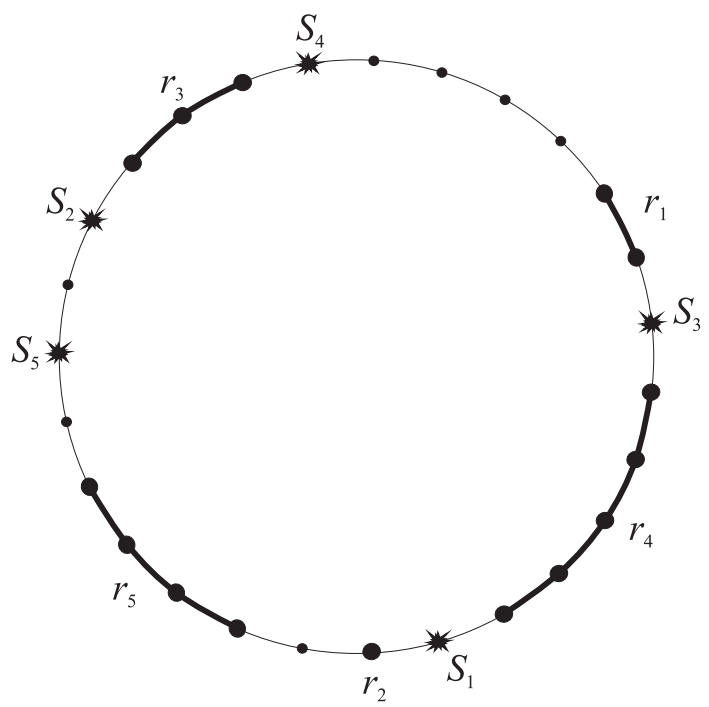

Figure 1: Battleship on a circle (Navy wins)

Lemma 1. In the games $G_{1}$ and $G_{2}$,

$$
\mathbf{P}\left\{\mathcal{D}_{1} \mathcal{D}_{2}\right\} \geqslant 1-\frac{4 q r-q^{2}-2 r+q}{M} .
$$

Proof. Let us start with $G_{1}$. Given $\left(r_{1}, \ldots, r_{q}\right)$, there are $M^{q}$ ways to deploy the fleet and exactly $M(q-1) !\left(\begin{array}{c}M-r+q-1 \\ q-1\end{array}\right)$ of these deployments avoid collisions. Indeed, each such deployment may be unambiguously reproduced as follows:

1) put the bow of the first ship at any of $M$ point on the circle;

2) permute remaining ships in one of $(q-1)$ ! ways;

3) choose $q$ non-negative intervals between successive ships starting from the first one. The tuple of intervals is a weak $q$-composition of $M-r$ and, therefore, may be chosen in $\left(\begin{array}{c}M-r+q-1 \\ q-1\end{array}\right)$ ways.

We repeat here the arguments of V. Nosov reported in [2]. The arguments 
yield:

$$
\begin{aligned}
\mathbf{P}\left\{\mathcal{D}_{1}\right\} & =\frac{M(q-1) !\left(\begin{array}{c}
M-r+q-1 \\
q-1
\end{array}\right)}{M^{q}}=\frac{(M-r+q-1)^{[q-1]}}{M^{q-1}} \\
& =\prod_{i=r-q+1}^{r-1}\left(1-\frac{i}{M}\right) \geqslant 1-\sum_{i=r-q+1}^{r-1} \frac{i}{M}=1-\frac{(2 r-q)(q-1)}{2 M} .
\end{aligned}
$$

Let $\overline{\mathcal{D}}_{2, i}$ be the event that the shot $S_{i}$ is successful. We have

$$
\mathbf{P}\left\{\overline{\mathcal{D}}_{2, i} \mid \mathcal{D}_{1}\right\}=\frac{r}{M}
$$

and, therefore,

$$
\begin{aligned}
\mathbf{P}\left\{\mathcal{D}_{2} \mid \mathcal{D}_{1}\right\}=1-\mathbf{P}\left\{\overline{\mathcal{D}}_{2,1} \cup \ldots \cup \overline{\mathcal{D}}_{2, q} \mid \mathcal{D}_{1}\right\} & \\
& \geqslant 1-\sum_{i=1}^{q} \mathbf{P}\left\{\overline{\mathcal{D}}_{2, i} \mid \mathcal{D}_{1}\right\}=1-\frac{q r}{M}
\end{aligned}
$$

In result,

$$
\begin{aligned}
\mathbf{P}\left\{\mathcal{D}_{1}\right\} \mathbf{P}\left\{\mathcal{D}_{2} \mid \mathcal{D}_{1}\right\} \geqslant\left(1-\frac{(2 r-q)(q-1)}{2 M}\right) & \left(1-\frac{q r}{M}\right) \\
& \geqslant 1-\frac{4 q r-q^{2}-2 r+q}{2 M},
\end{aligned}
$$

which was to be proven.

When passing from $G_{1}$ to $G_{2}$, the probability $\mathbf{P}\left\{\mathcal{D}_{1}\right\}$ does not decrease and we can use the just derived bound on this probability. The bound on $\mathbf{P}\left\{\mathcal{D}_{2} \mid \mathcal{D}_{1}\right\}$ also remains valid and we get the same resulting bound as for $G_{1}$.

An interesting question is what is the best strategy for an adversary in $G_{2}$. The partial answer is that with $q r \ll M$ the bound of Lemma 1 is almost reached when the adversary chooses $r_{1}=r-q+1, r_{2}=\ldots=r_{q}=1$ and shoots the circle with step $r_{1}$ starting from a random point. This tactic leads to the fact that to satisfy $\mathcal{D}_{1} \mathcal{D}_{2}$ the bow of the first ship must not occupy a continious segment of length $r_{1} q$. The second ship must avoid $r_{1}+q$ 
points, the third ship must avoid $r_{1}+q+1$ points and so on. In result,

$$
\begin{aligned}
\mathbf{P}\left\{\mathcal{D}_{1} \mathcal{D}_{2}\right\}=\left(1-\frac{r_{1} q}{M}\right) \prod_{i=1}^{q-1}\left(1-\frac{r_{1}+q+i-1}{M-i}\right) & \\
& \approx 1-\frac{r_{1} q}{M}-\sum_{i=1}^{q-1} \frac{r_{1}+q+i-1}{M} .
\end{aligned}
$$

The right part coincides with the bound of the lemma. Approximately the same probability will be achieved, if the adversary chooses $r_{1}=\ldots=$ $r_{q-1}=\lfloor r / q\rfloor$ and shoots again with step $r_{1}$.

\section{Security of CTR2}

To approve the security of CTR2, we use the standard notions sketched below (see [13] for further details and references).

1) An adversary (probabilistic algorithm) $A$ gains access to an encryption oracle $O$. The adversary interacts with $O$ using the following interface. It chooses a plaintext $X \in\{0,1\}^{*}$ and a nonce $S \in\{0,1\}^{n}$, sends to the oracle the pair $(X, S)$ and receives a ciphertext $Y \in\{0,1\}^{|X|}$. The adversary must use the interface following one of the two contracts: the nonces $S$ are either chosen uniformly independently at random (the random nonces contract) or they are arbitrary distinct (the nonrepeating nonces contract). Empty plaintexts are not allowed in both contracts.

2) The oracle $O$ may be implemented in two ways. In the first (real) implementation, the oracle actually performs the CTR2 encryption using a permutation $E_{K}$ chosen at random from $E$. This implementation is denoted by CTR2 $\left[E_{K}\right]$. In the second (ideal) implementation, the oracle picks $Y$ uniformly at random from $\{0,1\}^{|X|}$. This implementation is denoted by $\rho$.

3) The adversary sends to the oracle $O$ arbitrary queries, receives and analyzes corresponding answers. Its task is to distinguish the real implementation from ideal one. The adversary returns 1 (real) or 0 (ideal). Let $A^{O}$ be the output of $A$.

4) The distinguishing capabilities of $A$ are characterized by the advantage

$$
\mathbf{A d v}_{\mathrm{CTR} 2[E]}^{\text {ind-cpa }}(A)=\left|\mathbf{P}\left\{A^{\mathrm{CTR} 2\left[E_{K}\right]}=1\right\}-\mathbf{P}\left\{A^{\rho}=1\right\}\right| .
$$


The probabilities here are over the random tape of $A$ and over the random choice of $K$ and $\rho$. If $\mathbf{A d v}_{\mathrm{CTR} 2[E]}^{\text {ind-cpa }}(A)$ is small, then the two implementations are hard to distingush, which means the security of CTR2 based on $E$ relative to $A$. The used abbreviation ind-cpa covers the notion of indistinguishability and CPA settings: the adversary has access to the encryption oracle, but not to the decryption one.

Let us make a standard simplification replacing $E_{K}$, a random representative of $E$, with $\pi$, a random representative of $\operatorname{Perm}(n)$. This replacement turns $\operatorname{Adv}_{\mathrm{CTR} 2[E]}^{\text {ind-cpa }}(A)$ into the advantage

$$
\mathbf{A d v}_{\mathrm{CTR} 2[\operatorname{Perm}(n)]}^{\text {ind-cpa }}(A)=\left|\mathbf{P}\left\{A^{\mathrm{CTR} 2[\pi]}=1\right\}-\mathbf{P}\left\{A^{\rho}=1\right\}\right| .
$$

The replacement is motivated by the general assumption that permutations of a secure $E$ are hard to distinguish from random ones. The replacement is accompanied by a penalty (another advantage) which characterizes indistinguishability between random representativies of $E$ and $\operatorname{Perm}(n)$. This penalty is formal in nature (it is never estimated in practical cases), we do not specify it here for simplicity.

For given non-negative integers $q$ and $r, q \leqslant r$, we are interested in estimating

$$
\max _{A} \operatorname{Adv}_{\operatorname{CTR} 2[\operatorname{Perm}(n)]}^{\text {ind-cpa }}(A),
$$

where the maximum is taken over all adversaries that make $q$ queries to the oracle $O$ and the total length of plaintexts $X$ in these queries is equal to $r$. The length is specified in blocks, the last of which may be incomplete. Incomplete blocks of different plaintexts are counted separately.

The advantage of a reasonable $A$ does not increase if some full block is cut to incomplete. Therefore, we may assume without loss of the maximum advantage that all plaintexts and ciphertexts consist of full blocks.

Let us write again how CTR2 works, that is, how plaintexts $X_{1}, \ldots, X_{q}$ and nonces $S_{1}, \ldots, S_{q}$ are transformed into ciphertexts

$$
Y_{i}=\operatorname{CTR} 2[\pi]\left(X_{i}, S_{i}\right), \quad i=1, \ldots, q .
$$

Let $X_{i}$ consist of blocks $X_{i, 1}, \ldots, X_{i, r_{i}}, i=1, \ldots, q$, where $r_{i}>0$ and $r_{1}+$ $\ldots+r_{q}=r$. The corresponding blocks of the ciphertext $Y_{i}$ are

$$
Y_{i, j}=X_{i, j} \oplus \pi\left(C_{i, j}\right),
$$

where

$$
C_{i, 1}=\operatorname{next}^{c}\left(\pi\left(S_{i}\right)\right), \quad C_{i, 2}=\operatorname{next}\left(C_{i, 1}\right), \quad \ldots, \quad C_{i, r_{i}}=\operatorname{next}\left(C_{i, r_{i}-1}\right) .
$$


Here $c$ is an integer parameter of the mode. It equals 0 (the original CTR2) or 1 (GOST). In this section, the choice of $c$ is inessential. However, in the next section we use $c=1$.

Lemma 2. Let $N$ be a positive integer and $q, r$ be non-negative integers such that $q+r \leqslant N$. Then

$$
\frac{1}{(N-q)^{[r]}} \geqslant \frac{1}{N^{r}}\left(1+\frac{r(2 q+r-1)}{2 N}\right) .
$$

Proof. Consider three fractions: $1 /(N+2 q+r-1), 1 /(N-q-i)$ and $1 /(N-q-r+1+i), 0 \leqslant i \leqslant r-1$. The sum of their denominators is $3 N$. Therefore, the product of the denominators does not exceed $N^{3}$, the product of the fractions is not less than $1 / N^{3}$, and

$$
\frac{1}{N-q-i} \cdot \frac{1}{N-q-r+1+i} \geqslant \frac{N+2 q+r-1}{N^{3}}=\frac{1}{N^{2}}\left(1+\frac{2 q+r-1}{N}\right) .
$$

Hence,

$$
\left(\frac{1}{(N-q)^{[r]}}\right)^{2}=\prod_{i=0}^{r-1}\left(\frac{1}{N-q-i} \cdot \frac{1}{N-q-r+1+i}\right) \geqslant \frac{1}{N^{2 r}}\left(1+\frac{2 q+r-1}{N}\right)^{r},
$$

from which the result follows.

Theorem 1. Let $A$, an adversary against the n-bit block CTR2, make at most $q$ queries $(X, S)$ with nonempty $X$ and either random or nonrepeating $S$. Let $r$ be the total number of $X$ 's blocks in these queries. Let CTR2 be used with a permutation next whose largest cycle covers all blocks except possibly one. Then

$$
\operatorname{Adv}_{\operatorname{CTR} 2[\operatorname{Perm}(n)]}^{\text {ind-cpa }}(A) \leqslant \frac{r(r-1)}{2 N}+\varepsilon,
$$

where $N=2^{n}$ and (with the notation $(x)_{+}=\max (0, x)$ )

$$
\varepsilon=\left(\frac{2 q r-r+3 q+2}{2 N}-\frac{(r-q)^{2}}{2 N}+\frac{r(r+2 q-1)\left(6 q r-q^{2}-2 r+3 q+2\right)}{4 N^{2}}\right)_{+} .
$$

Proof. The bound easily holds for $q+r>N$ (in this case $r>N / 2$ ). Further we assume that $q+r \leqslant N$, so that Lemma 2 may be applied. We also note that $M$, the maximum cycle length of next, is at least $N-1$. 
Consider arbitrary nonempty plaintexts $X_{1}, \ldots, X_{q}, r$ full blocks in total, random or arbitrary non-repeating $S_{1}, \ldots, S_{q}$, and random $\pi, Y_{1}, \ldots, Y_{q}$. When we say random, we mean that implied objects are chosen uniformly at random from prescribed domains, each object independently of others.

Let the event $\mathcal{B}$ means that all $r$ blocks $\Gamma_{i, j}=X_{i, j} \oplus Y_{i, j}$ are distinct. For the complementary event $\overline{\mathcal{B}}$ it holds that

$$
\mathbf{P}\{\overline{\mathcal{B}}\} \leqslant \frac{r(r-1)}{2 N} .
$$

Let us introduce the probability

$$
p=\mathbf{P}\left\{\operatorname{CTR} 2[\pi]\left(X_{i}, S_{i}\right)=Y_{i}: i=1, \ldots, q \mid \mathcal{B}\right\}
$$

and apply Patarin's "coefficients $H$ " technique (see [12] and also $[4,5,11]$ ). According to this technique, if the inequality $p \geqslant(1-\varepsilon) / N^{r}$ with some $\varepsilon \geqslant 0$ holds, then the desired advantage is upper bounded by the sum $\mathbf{P}\{\overline{\mathcal{B}}\}+\varepsilon$. It remains to prove that $\varepsilon$ from the statement of the theorem indeed satisfies the inequality.

Consider the following events, each new one provided that previous events occur.

The event $\mathcal{C}$ : all blocks $\pi\left(S_{i}\right)$ fall into the largest cycle of next. The probability $p_{C}=\mathbf{P}\{\mathcal{C} \mid \mathcal{B}\}$ is obviously 1 if $M=N$. For $M=N-1$ it equals either $M^{q} / N^{q}$ in the case of random nonces or $M^{[q]} / N^{[q]}$ in the case of non-repeating nonces. In both cases,

$$
p_{C} \geqslant \frac{M}{N}\left(1-\frac{q}{N}\right) \text {. }
$$

Indeed,

$$
\frac{M^{q}}{N^{q}} \geqslant \frac{M^{[q]}}{N^{[q]}}=\frac{M}{N} \cdot \frac{(M-1)^{[q-1]}}{(N-1)^{[q-1]}}=\frac{M}{N} \cdot \frac{M-q+1}{N-1} \geqslant \frac{M}{N} \cdot \frac{N-q}{N-1} \geqslant \frac{M}{N}\left(1-\frac{q}{N}\right) .
$$

The event $\mathcal{D}$ : all counters $C_{i, j}$ (they are all on the largest cycle according to $\mathcal{C}$ ) differ from each other and from nonces $S_{k}$. The probability of this event is already estimated in Lemma 1 of the previous section:

$$
p_{D}=\mathbf{P}\{\mathcal{D} \mid \mathcal{B C}\} \geqslant 1-\frac{4 q r-q^{2}-2 r+q}{2 M} .
$$

We indeed satisfy the rules of the game described there, if we imagine that the initial counters $C_{i, 1}$ are placed on the cycle randomly and after 
that, in the case of no collisions, the random permutation $\pi$ either "generates" random distinct $S_{i}=\pi^{-1}\left(\right.$ next $^{-c}\left(C_{i, 1}\right)$ ) or implicitly transfers the given distinct $S_{i}$ into next ${ }^{-c}\left(C_{i, 1}\right)$. It may be that some $S_{i}$ lies outside the cycle. In this case, the probability $p_{D}$ only increases with respect to the probability treated in Lemma 1 and the bound of the lemma remains valid.

Consider the probability $p_{C D}=\mathbf{P}\{\mathcal{C D} \mid \mathcal{B}\}=p_{C} p_{D}$. Dealing with the case $M=N-1$, we get

$p_{C D} \geqslant\left(1-\frac{q}{N}\right)\left(\frac{M}{N}-\frac{4 q r-q^{2}-2 r+q}{2 N}\right) \geqslant 1-\frac{4 q r-q^{2}-2 r+3 q+2}{2 N}$.

Obviously, this bound also holds for $M=N$.

The event $\mathcal{E}$ : no collisions $\Gamma_{i, j}=\pi\left(S_{k}\right)$ occur. There are $q r$ possible collisions, each occurs with probability $1 / N$ and, therefore,

$$
p_{E}=\mathbf{P}\{\mathcal{E} \mid \mathcal{B C D}\} \geqslant 1-\frac{q r}{N} .
$$

The event $\mathcal{F}: \pi$ maps $C_{i, j}$ to $\Gamma_{i, j}$. The previous events means that all $\Gamma_{i, j}$ are distinct, all $C_{i, j}$ are distinct, all $S_{i}$ are distinct, $C_{i, j}$ differ from $S_{k}$, $q$ images of $\pi$ at points $S_{k}$ are already known and they differ from $\Gamma_{i, j}$. So there are $(N-q)$ ! ways to determine remaining images of $\pi$ and exactly $(N-q-r)$ ! of them are in favor of $\mathcal{F}$. Therefore,

$$
p_{F}=\mathbf{P}\{\mathcal{F} \mid \mathcal{B C D} \mathcal{E}\}=\frac{1}{(N-q)^{[r]}} \geqslant \frac{1}{N^{r}}\left(1+\frac{r(r+2 q-1)}{2 N}\right) .
$$

Here we use Lemma 2.

In result,

$$
\begin{aligned}
p & \geqslant \mathbf{P}\{\mathcal{C D} \mathcal{E} \mathcal{F} \mid \mathcal{B}\}=p_{C D} p_{E} p_{F} \\
& \geqslant \frac{1}{N^{r}}\left(1-\frac{4 q r-q^{2}-2 r+3 q+2}{2 N}\right)\left(1-\frac{q r}{N}\right)\left(1+\frac{r(r+2 q-1)}{2 N}\right) \\
& \geqslant \frac{1}{N^{r}}\left(1-\frac{6 q r-q^{2}-2 r+3 q+2}{2 N}\right)\left(1+\frac{r(r+2 q-1)}{2 N}\right)
\end{aligned}
$$

from which the expression for $\varepsilon$ follows.

It is easy to verify that $\varepsilon$ increases as a function of $q$ for $q \leqslant r$. Replacing $q$ with $r$ in $\varepsilon$, we obtain the following bound, uniform in $q$ :

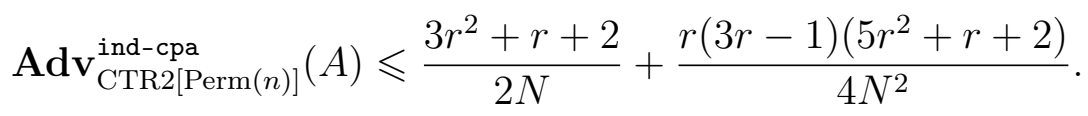


For comparison, a similar advantage for the CTR mode is upper bounded by $r^{2} /(2 N)$ (see [13]). Informally, in the region $r^{2} \ll N$, which is used in practice, the transition from CTR to CTR2 is accompanied by a penalty, the main contribution to which is made by the term $r^{2} / N$.

In the just considered case $q=r$, the average length $\mu$ of plaintexts measured in blocks equals 1 . This case is the most inefficient, it requires 2 invocations of block encryption per block of processed data. If we increase $\mu$, we will not only speed up encryption but also improve our bound on advantage in terms of $r$. For example, with $\mu=2$ the main term in the bound is $7 r^{2} /(8 N)$ and with $\mu=3$ it is $11 r^{2} /(18 N)$. The main term takes the form $r^{2} /(2 N)$, that is, we achieve the CTR bound as soon as $\mu$ becomes larger than $2+\sqrt{3} \approx 3.73$.

\section{CHE and its security}

In this section, we extend CTR2 to the authentication encryption mode called CHE (Counter + Hash + Encrypt). The extended functionality of CHE is data authentication. CHE follows the Encrypt-then-MAC paradigm (first encrypt, then authenticate) which seems to be better than the MAC-thenEncrypt alternative (see [3]). Not only encrypted data is authenticated, but also associated data that is transmitted in the plain form. Thus, CHE belongs to the AEAD (Authentication Encryption with Associated Data) class of the AE schemes.

Let us interpret blocks of $\{0,1\}^{n}$ as elements of the finite field $\mathbb{F}$ of order $N$. Suppose that the usual correspondence between $\mathbb{F}$ and $\{0,1\}^{n}$ is used, when the addition in $\mathbb{F}$ is $\oplus$. Let

$$
\operatorname{next}(\lambda)=a * \lambda \oplus b
$$

where $a$ is a primitive element of $\mathbb{F}, b$ is a nonzero element. Hereinafter we make the multiplication sign explicit. As we have already noted, the maximum cycle length of next is $N-1$. Moreover, the powers next ${ }^{i}, i=$ $1,2, \ldots, N-2$, considered as polynomials over $\mathbb{F}$ all have nonzero constant terms.

The CHE mode is determined by the algorithms WRAP (encrypt and generate the authentication tag) and UNWRAP (verify the tag and decrypt) defined in Fig. 2. The inputs and outputs of the algorithms are: a plaintext $X \in\{0,1\}^{*}$, associated data $I \in\{0,1\}^{*}$, a key $K \in \mathcal{K}$, a nonce $S \in\{0,1\}^{*}$, a ciphertext $Y \in\{0,1\}^{|X|}$, an authentication tag $T \in\{0,1\}^{n}$. An arbitrary nonzero $T_{0} \in\{0,1\}^{n}$ is used. The operation $\stackrel{n}{\leftarrow}$ means splitting 
a binary word into $n$-bit blocks preceded by padding to the block size. The reverse operation $\leftarrow$ means assembling a word from several blocks followed by truncation to $m$ bits.

\begin{tabular}{|c|c|}
\hline Algorithm WRAP & Algorithm UNWRAP \\
\hline Input: $X, I, K, S$. & Input: $Y, I, K, S, T$. \\
\hline Output: $Y, T$. & Output: $X$ or $\perp$ (authentication error). \\
\hline Steps: & Steps: \\
\hline 1) $H \leftarrow E_{K}(S), C \leftarrow H, T \leftarrow T_{0}$. & 1) $H \leftarrow E_{K}(S), C \leftarrow H, T^{\prime} \leftarrow T_{0}$. \\
\hline 2) $\left(I_{1}, \ldots, I_{r^{\prime}}\right) \stackrel{n}{\leftarrow} I$. & 2) $\left(I_{1}, \ldots, I_{r^{\prime}}\right) \stackrel{n}{\leftarrow} I$. \\
\hline 3) For $i=1,2, \ldots, r^{\prime}$ : & 3) For $i=1,2, \ldots, r^{\prime}$ : \\
\hline a) $T \leftarrow\left(T \oplus I_{i}\right) * H$ & a) $T^{\prime} \leftarrow\left(T^{\prime} \oplus I_{i}\right) * H$ \\
\hline 4) $\left(X_{1}, \ldots, X_{r}\right) \stackrel{n}{\leftarrow} X$. & 4) $\left(Y_{1}, \ldots, Y_{r}\right) \stackrel{n}{\leftarrow} Y$. \\
\hline 5) For $i=1,2, \ldots, r$ : & 5) For $i=1,2, \ldots, r$ : \\
\hline a) $C \leftarrow \operatorname{next}(C)$ & a) $T^{\prime} \leftarrow\left(T^{\prime} \oplus Y_{i}\right) * H$ \\
\hline b) $Y_{i} \leftarrow X_{i} \oplus E_{K}(C)$ & b) $C \leftarrow \operatorname{next}(C)$ \\
\hline c) $T \leftarrow\left(T \oplus Y_{i}\right) * H$ & c) $X_{i} \leftarrow Y_{i} \oplus E_{K}(C)$. \\
\hline 6) $Y \underset{|X|}{\overleftarrow{t} \mid}\left(Y_{1}, \ldots, Y_{r}\right)$ & 6) $X \underset{|Y|}{\overleftarrow{t} \mid}\left(X_{1}, \ldots, X_{r}\right)$ \\
\hline 7) Encode $|I|$ and $|X|$ by $W \in\{0,1\}^{n}$. & 7) Encode $|I|$ and $|X|$ by $W \in\{0,1\}^{n}$. \\
\hline 8) $T \leftarrow(T \oplus W) * H$ & 8) $T^{\prime} \leftarrow\left(T^{\prime} \oplus W\right) * H$ \\
\hline 9) $T \leftarrow E_{K}(T)$. & 9) $T^{\prime} \leftarrow E_{K}\left(T^{\prime}\right)$. \\
\hline 10) Return $(Y, T)$. & $\begin{array}{l}\text { 10) Return } X \text { if } T=T^{\prime} \text { and } \perp \text { other- } \\
\text { wise. }\end{array}$ \\
\hline
\end{tabular}

Figure 2: The CHE mode $($ next $(C)=a * C \oplus b)$

It is assumed that in Step 7 of both algorithms, different pairs $(|I|,|X|)$ give different words $W$, and $W$ is zero if and only if $|I|=|X|=0$.

The algorithm WRAP may be explained in the following way.

C. First, the CTR2 encryption is performed: $Y \leftarrow \operatorname{CTR} 2\left[E_{K}\right](X, S)$. The encrypted nonce $H=E_{K}(S)$ is used to build internal counters $\operatorname{next}^{i}(H), i=1,2, \ldots$

H. Second, a polynomial $f_{(Y, I)}(\lambda) \in \mathbb{F}[\lambda]$ is implicitly constructed from the pair $(Y, I)$. This polynomial has a positive degree, its constant term equals 0 , different pairs give different polynomials. The polynomial is evaluated at the point $H$, the result $Z=f_{(Y, I)}(H)$ becomes a hash value of $(Y, I)$.

E. Third, the hash value $Z$ is encrypted and returned as $T$ along with $Y$. 
Suppose that $\operatorname{deg} f_{(Y, I)} \leqslant d$. In other words, at most $d-1$ blocks of $I$ and $Y$ are processed during a single invocation of polynomial hashing. Suppose further that $d<N-1$. The restrictions on structure and degree of the polynomials $f_{(Y, I)}$ and the form of next lead to the following estimates (see [1] for details):

$$
\left.\begin{array}{l}
\mathbf{P}\left\{f_{(Y, I)}(H)=f_{\left(Y^{\prime}, I^{\prime}\right)}\left(H^{\prime}\right)\right\} \\
\mathbf{P}\left\{f_{(Y, I)}(H)=f_{\left(Y^{\prime}, I^{\prime}\right)}\left(H^{\prime}\right) \mid H \neq H^{\prime}\right\} \\
\mathbf{P}\left\{f_{(Y, I)}(H)=h\right\} \\
\mathbf{P}\left\{f_{(Y, I)}(H)=\operatorname{next}^{i}(H)\right\} \\
\mathbf{P}\left\{f_{(Y, I)}(H)=\operatorname{next}^{i}\left(H^{\prime}\right) \mid H \neq H^{\prime}\right\}
\end{array}\right\} \leqslant \frac{d}{N} .
$$

Here $1 \leqslant i \leqslant d, h$ is a fixed element of $\mathbb{F}$, the probabilities are taken over independent random $H, H^{\prime} \in \mathbb{F}$. These estimates form the basis for justifying the security of CHE.

Dealing with the security, we keep the model introduced in the previous section. An adversary interacts with an oracle $O:(X, I, S) \mapsto(Y, T)$ which either implements the WRAP algorithm (the real implementation, $\mathrm{CHE}\left[E_{K}\right]$ ) or generates $Y \in\{0,1\}^{|X|}$ and $T \in\{0,1\}^{n}$ at random (the ideal implementation, $\rho$ ). The adversary again follows one of the two contracts: random nonces or non-repeating nonces. Each of the words $X$ and $I$ may be empty.

An advantage of the adversary is defined in the standard way. We only change the abbreviation ind-cpa to priv (privacy). This corresponds to the tradition when moving from conventional encryption to AEAD.

We again idealize $E$ and replace its representative $E_{K}$ with a permutation $\pi$ chosen uniformly at random from $\operatorname{Perm}(n)$.

Theorem 2. Let $A$, an adversary against the $n$-bit block CHE, make at most $q$ queries $(X, I, S)$ with either random or non-repeating $S$. Let $r$ be the total number of $X$ 's blocks in these queries. Let the total number of blocks in each pair $(X, I)$ be less then $d$. Then

$$
\operatorname{Adv}_{\operatorname{CHE}[\operatorname{Perm}(n)]}^{\text {priv }}(A) \leqslant \frac{(r+q)(r+q-1)}{2 N}+\alpha-\beta+\alpha \beta,
$$

where $N=2^{n}$ and

$\alpha=\frac{1}{2 N}\left((2 d+6) q r+(3 d+5) q^{2}-2 r-(d-3) q+2\right), \beta=\frac{1}{2 N}(r+q)(r+3 q-1)$. 
Proof. We adapt the proof of Theorem 1 preserving notations and following the general line. Additional notations: $I_{i}$ - associated data in the $i$-th query, $T_{i}$ - a tag in the $i$ th answer, $H_{i}=\pi\left(S_{i}\right), Z_{i}=f_{\left(Y_{i}, I_{i}\right)}\left(H_{i}\right)$. Recall that we allow plaintexts $X_{i}$ to be empty. Let $q_{1}$ be the total number of queries with nonempty plaintexts.

If $d \geqslant N-1$ or $r+2 q>N$, then the bound of the theorem is easily satisfied. Further we assume that $d<N-1$ and $r+2 q \leqslant N$.

We preserve the probabilistic model of Theorem 1 assuming additionally that $T_{i}$ are chosen uniformly independently at random. Now the event $\mathcal{B}$ additionally means that $T_{i}$ are distinct and different from $\Gamma_{j, k}$. We have

$$
\mathbf{P}\{\overline{\mathcal{B}}\} \leqslant \frac{(r+q)(r+q-1)}{2 N} .
$$

For the probability

$$
p=\mathbf{P}\left\{\operatorname{CHE}[\pi]\left(X_{i}, I_{i}, S_{i}\right)=\left(Y_{i}, T_{i}\right): i=1, \ldots, q \mid \mathcal{B}\right\},
$$

it is necessary to construct the inequality $p \geqslant(1-\varepsilon) / N^{r+q}$ with $\varepsilon=$ $\alpha-\beta+\alpha \beta$. To do this, we deal again with the events $\mathcal{C}, \mathcal{D}, \mathcal{E}$ and $\mathcal{F}$.

The semantics of $\mathcal{C}$ and $\mathcal{D}$ are not changed. We require that the sequences of counters lie on the largest cycle of next, that the sequences do not intersect (hence the corresponding nonces do not collide), and that the sequences do not cover nonces. We treat $q_{1}$ sequences and nonces that correspond to nonempty plaintexts and have

$$
p_{C D} \geqslant 1-\frac{4 q_{1} r-q_{1}^{2}-2 r+3 q_{1}+2}{2 N} .
$$

In $\mathcal{E}$ we block the following collisions:

\begin{tabular}{|c|c|c|}
\hline collisions & quantity & probability \\
\hline \hline$Z_{i}=Z_{j}$ & $q(q-1) / 2$ & $\leqslant d / N$ \\
$Z_{i}=S_{j}$ & $q^{2}$ & $\leqslant d / N$ \\
$Z_{i}=C_{j, k}$ & $q r$ & $\leqslant d / N$ \\
$H_{i}=\Gamma_{j, k}$ & $q r$ & $1 / N$ \\
$H_{i}=T_{j}$ & $q^{2}$ & $1 / N$ \\
\hline
\end{tabular}

In addition, we require that $q-q_{1}$ nonces $S_{i}$ that correspond to empty plaintexts are pairwise distinct and differ from each of the remaining $q_{1}$ nonces and each of the counters. Thus, we block $\left(q-q_{1}\right)\left(q-q_{1}-1\right) / 2+$ $\left(q-q_{1}\right) q_{1}$ collisions of the form $S_{i}=S_{j}$ (note that they are automatically 
blocked if nonces are not repeated) and $\left(q-q_{1}\right) r$ collisions of the form $S_{i}=C_{j, k}$. Collisions occur with probabilities $\leqslant 1 / N$.

With this,

$p_{E} \geqslant 1-\frac{(2 d+2) q r+(3 d+2) q^{2}-d q+2\left(q-q_{1}\right) r+\left(q-q_{1}\right)\left(q-q_{1}-1\right)+2\left(q-q_{1}\right) q_{1}}{2 N}$

and $p_{C D} p_{E} \geqslant 1-\alpha_{0}$, where

$$
\alpha_{0}=\frac{1}{2 N}\left((2 d+4) q r+(3 d+3) q^{2}-2 r-(d+1) q+(2 r+4) q_{1}-2 q_{1}^{2}+2\right) .
$$

The quantity $\alpha_{0}$ as a function of $q_{1} \in[0, q]$ attains its maximum at either $q_{1}=r / 2+1$ or $q_{1}=q$. The maximum is

$$
\frac{1}{2 N} \begin{cases}(2 d+4) q r+(3 d+3) q^{2}-2 r-(d+1) q+2+2(r / 2+1)^{2}, & r / 2+1 \leqslant q, \\ (2 d+6) q r+(3 d+1) q^{2}-2 r-(d-3) q+2, & r / 2+1>q .\end{cases}
$$

In either case, the maximum is not larger than $\alpha$.

In $\mathcal{F}$ we require that $\pi$ not only maps $C_{i, j}$ to $\Gamma_{i, j}$, but also maps $Z_{i}$ to $T_{i}$. The previous events mean that all preimages here are pairwise distinct, all images are pairwise distinct, $q$ images of $\pi$ at points $S_{i}$ that differ from $C_{j, k}$ and $Z_{j}$ are already known and these images differ from $\Gamma_{j, k}$ and $T_{j}$. The total number of preimages is $r+q$. Therefore,

$$
p_{F} \geqslant \frac{1}{(N-q)^{[r+q]}} \geqslant \frac{1}{N^{r+q}}(1+\beta) .
$$

In result,

$$
p \geqslant p_{C D} p_{E} p_{F} \geqslant \frac{1}{N^{r+q}}(1-\alpha+\beta-\alpha \beta),
$$

which was to be proven.

From the perspective of the theorem, to provide security guarantees, CHE must be used with $r$ and $d q$ much smaller than $\sqrt{N}$. With such quotas the main contribution to the bound on the adversary's advantage is made by the terms

$$
\frac{1}{2 N}\left((2 d+4) q r+(3 d+3) q^{2}\right) .
$$

Acknowledgement: The author thanks the anonymous referees of CTCRYPT 2019 and of the journal for their valuable feedback. 


\section{References}

[1] Agievich S., "EHE: nonce misuse-resistant message authentication", Prikl. Discr. Mat., 39 (2018), 33-41, https://eprint.iacr.org/2017/231.

[2] Babash A.V., Shankin G.P., Cryptography, Solon-Press, Moscow, 2007 (In Russian).

[3] Bellare M., Namprempre C., "Authenticated encryption: Relations among notions and analysis of the generic composition paradigm", J. Cryptology, 21:4 (2008), 469-491.

[4] Bernstein D., "A short proof of the unpredictability of cipher block chaining", 2005, http://cr.yp.to/papers.html\#easycbc.

[5] Chen S., Steinberger J., "Tight security bounds for key-alternating ciphers", EUROCRYPT 2014, Lect. Notes Comput. Sci., 8441, 2014, 327-350.

[6] Dworkin M., NIST SP 800-38A. Recommendation for Block Cipher Modes of Operation: Methods and Techniques, National Institute of Standards and Technology (NIST) of the U.S., 2001.

[7] GOST 28147-89. Cryptographic Protection for Data Processing Systems. Goverment Standard of the USSR, IPK Izd-vo standartov, Moscow, 1989 (In Russian).

[8] GOST R 34.13-2015. Information technology. Cryptographic data security. Block ciphers operation modes. Government Standard of the Russian Federation, Standardinform, Moscow, 2015 (In Russian).

[9] ISO/IEC 10116. Information technology - Security techniques - Modes of operation of an n-bit cipher. Third edition, 2006.

[10] McGrew D.A., Viega J., "The security and performance of the Galois/Counter Mode (GCM) of operation", INDOCRYPT 2004, Lect. Notes Comput. Sci., 3348, 2005, 343-355.

[11] Nandi M., "Improved security analysis for OMAC as a pseudorandom function", J. Math. Cryptol., 3 (2009), 133-148.

[12] Patarin J., Etude des Gènèrateurs de Permutations Basès sur le Sch'ema du D.E.S., Thèse de Doctorat en Sci. Appl., de l'Univ. de Paris 6, 1991 (In French).

[13] Rogaway P., "Evaluation of some blockcipher modes of operation", Cryptography Research and Evaluation Committees (CRYPTREC), 2011, http://www.cryptrec.go.jp/estimation/techrep_id2012_2.pdf.

[14] Rogaway P., "Nonce-based symmetric encryption", FSE 2004, Lect. Notes Comput. Sci., 3017, 2004, 348-358.

[15] STB 34.101.31-2011. Information Technology and Security. Data Encryption and Integrity Algorithms. Standard of Belarus, 2011 (In Russian), http://apmi.bsu.by/assets/files/std/belt-spec27.pdf. 